\title{
Heart rate turbulence in patients with poorly controlled diabetes mellitus type 2
}

\author{
Andrzej Bissinger, Jan Ruxer, Rehana B. Ahmed, Andrzej Lubinski
}

Department of Invasive Cardiology and Cardiodiabetology, Medical University of Lodz, Lodz, Poland

Submitted: 16 September 2012

Accepted: 26 January 2013

Arch Med Sci 2014; 10, 6: 1073-1077

DOI: $10.5114 /$ aoms.2014.47819

Copyright $\odot 2014$ Termedia \& Banach

\section{Abstract}

Introduction: Cardiac autonomic neuropathy (CAN) causes substantial morbidity and increased mortality in patients with diabetes mellitus (DM). Besides heart rate variability (HRV), heart rate turbulence (HRT) is an important method of assessment of cardiac autonomic regulation. The aim of the study was to assess the correlation between HRT and diabetic control.

Material and methods: Fifty-nine patients met the inclusion criteria - 38 males and 21 females, age $64.4 \pm 7.6$. The patients included had inadequately controlled DM type 2 defined as glycated haemoglobin $\left(\mathrm{HbA}_{1 \mathrm{c}}\right)>9 \%$ (mean 11.8 $\pm 2.7 \%$ ). In all patients, intensive insulin treatment had been applied for 6 months. After 6 months, $\mathrm{HbA}_{1 \mathrm{c}}$ was measured. ECG Holter monitoring was performed before and after insulin treatment to evaluate the time domain HRV and HRT parameters (turbulence onset (TO) and turbulence slope (TS)). Results: After 6 months of intensive insulin treatment, $\mathrm{HbA}_{1 \mathrm{c}}$ concentrations ranged from $6.3 \%(45 \mathrm{mmol} / \mathrm{mol})$ to $11.2 \%(99 \mathrm{mmol} / \mathrm{mol})-$ mean $8.5 \pm 3.8 \%$ $(69 \pm 18 \mathrm{mmol} / \mathrm{mol})$. Significant improvement of TO, TS and SDNN was observed. The TO and TS significantly correlated with $\mathrm{HbA}_{1 c}(r=0.35, p=0.006$ and $r=-0.31, p=0.02$ respectively). Among analyzed HRV time domain parameters such as SDNN, rMSSD and pNN50, only SDNN correlated with $\mathrm{HbA}_{1 c}(r=-0.41, p=0.001)$. It was further concluded that intensive insulin therapy led to better glycemic control, resulting in improvement of HRT.

Conclusions: Heart rate turbulence may be useful in monitoring changes of the autonomic nervous system functions in patients with DM, similarly to HRV parameters.

Key words: diabetes mellitus, heart rate turbulence, diabetic autonomic neuropathy.

\section{Introduction}

Diabetic autonomic neuropathy causes substantial morbidity and increases mortality, more specifically if cardiovascular autonomic neuropathy (CAN) is present. A patient's history and physical examination are ineffective for early detection of CAN. Therefore noninvasive tests that have demonstrated efficacy are vital to diagnose and detect CAN earlier, rather than later [1]. Heart rate turbulence (HRT) has become an important method of assessment of cardiac autonomic regulation. It is a reliable indicator of baroreceptor sensitivity [2,3]. Previous studies have revealed decreased HRT parameters in diabetes mellitus type 2 , with and without cardiac autonomic neuropathy $[4,5]$. Other studies have shown

\author{
Corresponding author: \\ Dr. Andrzej Bissinger \\ Department of Invasive \\ Cardiology and \\ Cardiodiabetology \\ Medical University of Lodz \\ 113 Zeromskiego St \\ 90-549 Lodz, Poland \\ Phone: +48 426393563 \\ Phone/fax: +48 426393561 \\ Fax: +48 426393563 \\ E-mail: abissinger@gmail. \\ com
}


that strict glycemic control leads to improvement of the autonomic nervous system $[6,7]$.

The aim of this study was to determine the influence of glycemic control on HRT.

\section{Material and methods}

Fifty-nine patients with poorly controlled diabetes mellitus type 2 met the criteria for inclusion in the study. Hemoglobin $\mathrm{A}_{1 \mathrm{c}}$ concentration $\left(\mathrm{HbA}_{1 \mathrm{c}}\right)$ was used to assess glycemic control. Patients with $\mathrm{HbA}_{1 \mathrm{c}}>9 \%$ and with ventricular premature beats (VPBs) agreeing to HRT analysis were used in the study. All participants underwent a 24-hour Holter recording (SUPRIMA 12, DMS, USA) to assess time domain HRV and HRT components, turbulence onset (TO) and turbulence slope (TS). All Holter recordings were performed in an ambulatory setting. Recordings from digital 12-lead recorders (DMS 300-12, USA), with a sample rate of 1024 bps, were analyzed. The initial heart rhythm acceleration after ventricular premature beat is defined as TO and the ensuring deceleration as TS. Recordings lasting for $\geq 18 \mathrm{~h}$ containing at least 30 premature ventricular beats were included in the analysis. According to HRT consensus guidelines [8], sequences with at least 2 sinus rhythm R-R intervals before VPBs, and at least 15 subsequent sinus R-R intervals were included in the HRT analysis. Also analyzed VPBs were limited to VPBs with prematurity $>20 \%$ and a compensatory pause of $>120 \%$ of the mean of the last five sinus rhythm intervals preceding the VPB. After manual review of the Holter tracings, TO and TS were determined according to a previously published method [8]. In cases in which the criterion was not achieved, recordings were repeated - maximally three times. If Holter recordings were non-interpretable despite three rep-

Table I. Baseline characteristics of patients included in the study

\begin{tabular}{|lc|}
\hline Parameter & Results \\
\hline Number of patients & 59 \\
\hline Sex (male/female) & $38 / 21$ \\
& $(64 \% / 36 \%)$ \\
\hline Age, mean \pm SD [years] & $64.4 \pm 7.6$ \\
\hline Body mass index, mean \pm SD [kg/m²] & $30.3 \pm 4.5$ \\
\hline Duration of diabetes, mean \pm SD [years] & $5.0 \pm 1.3$ \\
\hline Baseline HbA ${ }_{1 c}$ mean \pm SD [\%, mmol/mol] & $11.8 \pm 2.7$, \\
& $105 \pm 13$ \\
\hline Insulin therapy, $n$ (\%) & $15(25)$ \\
\hline Oral antidiabetic drugs, $n$ (\%) & $35(59)$ \\
\hline Insulin + oral antidiabetic drug, $n(\%)$ & $5(8)$ \\
\hline No diabetes treatment or diet only, $n(\%)$ & $4(7)$ \\
\hline
\end{tabular}

\section{Results}

Characteristics of studied patients are listed in Table I.

In the study group, baseline $\mathrm{HbA}_{1 \mathrm{c}}$ concentration was $11.8 \pm 2.7 \%(105 \pm 13 \mathrm{mmol} / \mathrm{mol})$. After 6 months of intensive insulin treatment, mean $\mathrm{HbA}_{1 \mathrm{c}}$ concentration was $8.5 \pm 3.8 \%(69 \pm 18 \mathrm{mmol} / \mathrm{mol})$. Significant improvement of TO, TS and standard deviation of all normal-normal intervals (SDNN) were also observed, as presented in Table II. Examples of HRT parameter changes in the single patient are shown in Figures 1 and 2 .

At the end of the study, the HRT parameters TO and TS correlated with $\mathrm{HbA}_{1 \mathrm{c}}(r=0.35, p=0.006$ and $r=-0.31, p=0.02$ respectively) (Figures 3 and 4).

Among the analyzed HRV time domain parameters SDNN, square root of the mean of the squares of differences between adjacent normal-normal 
Table II. Changes of HRT and HRV after intensive insulin treatment

\begin{tabular}{|lccc|}
\hline Parameter & Before intensive insulin therapy & After intensive insulin therapy & Value of $p$ \\
\hline TO $(\%)$ & $-1.1 \pm 1.4$ & $-1.6 \pm 1.4$ & 0.001 \\
\hline TS [ms/RR] & $12.1 \pm 9.5$ & $13.8 \pm 6.7$ & 0.01 \\
\hline SDNN [ms] & $113.7 \pm 16.2$ & $119.5 \pm 14.9$ & 0.001 \\
\hline rMSSD [ms] & $22.9 \pm 8.8$ & $23.4 \pm 8.5$ & NS \\
\hline pNN50 (\%) & $3.5 \pm 1.4$ & $3.6 \pm 1.5$ & NS \\
\hline
\end{tabular}

TO - turbulence onset, TS - turbulence slope, SDNN - standard deviation of all normal-normal intervals, rMSSD - square root of the mean of the squares of differences between adjacent normal-normal intervals, pNN50 - percentage of differences between adjacent normalnormal intervals greater than $50 \mathrm{~ms}$.

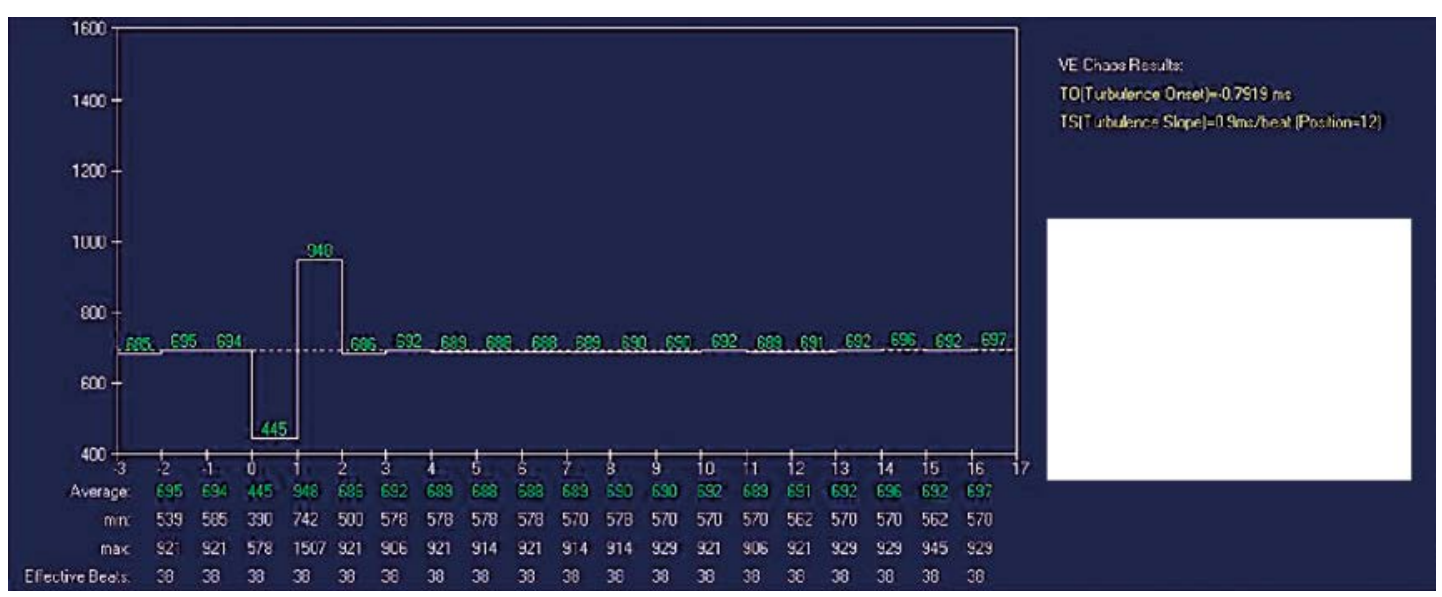

Figure 1. Example of heart rate turbulence (HRT) analysis in a patient before intensive insulin treatment. Turbulence onset $(\mathrm{TO})=0.7919 \mathrm{~ms}$, turbulence slope $(\mathrm{TS})=0.9 \mathrm{~ms} / \mathrm{RR}$

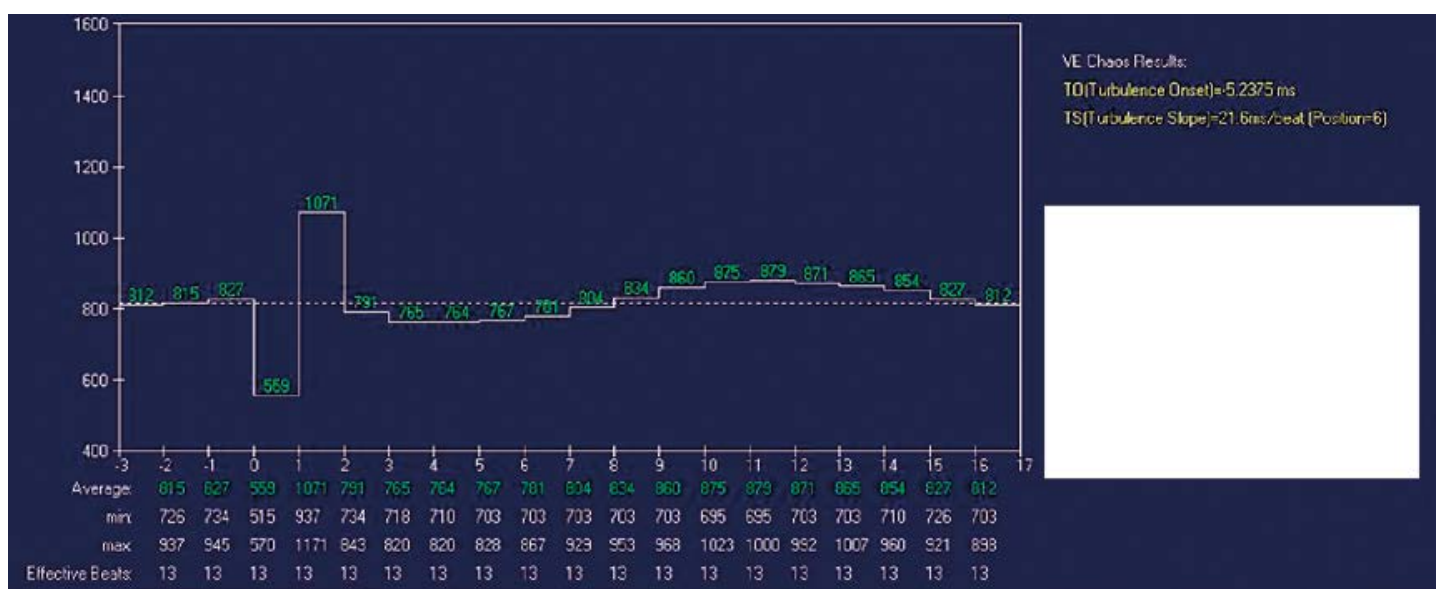

Figure 2. Example of heart rate turbulence (HRT) analysis in the same patient as presented at Figure 1, after intensive insulin treatment. Turbulence onset $(\mathrm{TO})=-5.2375 \mathrm{~ms}$, turbulence slope $(\mathrm{TS})=21.6 \mathrm{~ms} / \mathrm{RR}$

intervals (rMSSD) and percentage of differences between adjacent normal-normal intervals greater than 50 ms ( $p N N 50)$, only SDNN correlated with $\mathrm{HbA}_{1 \mathrm{c}}(r=-0.41, p=0.001)$ (Figure 5).

\section{Discussion}

The main finding of this study is that improvement of glycemic control leads to improvement of HRT parameters. Heart rate turbulence is an indirect measurement of baroreflex sensitivity depen- dent on autonomic nervous system function. The metabolic disorder of diabetes leads to damage of peripheral and autonomic nerves. Clinical manifestations of autonomic dysfunction frequently occur concurrently but not in consistent patterns. Therefore, it can be suspected that a patient diagnosed with diabetes mellitus type 2 should have at least subclinical disturbances of the autonomic nervous system [9]. Cardiovascular autonomic neuropathy has been linked to cardiac arrhythmias, postural hypotension, exercise intolerance, 


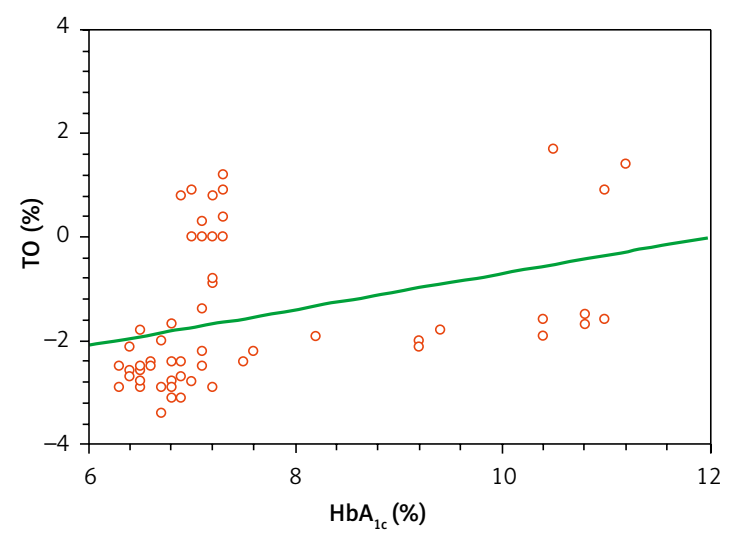

Figure 3. Correlation between TO (turbulence onset) and $\mathrm{HbA}_{1 \mathrm{c}}$ concentration $(r=0.35, p=0.006)$

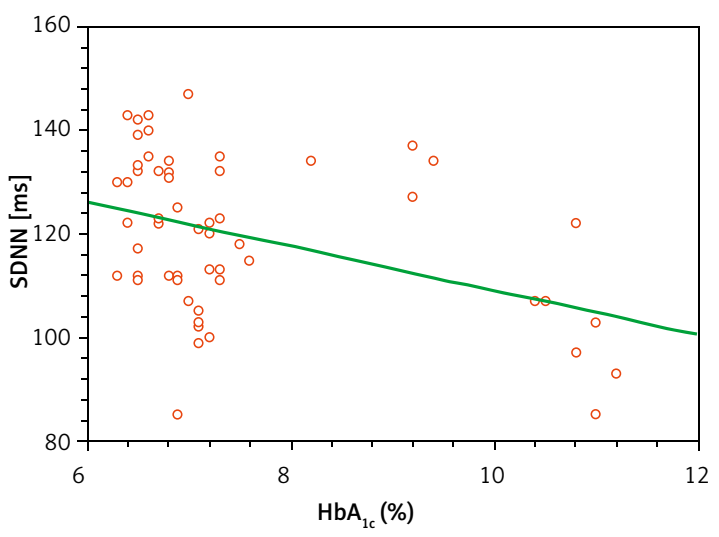

Figure 5. Correlation between $\mathrm{SDNN}$ and $\mathrm{HbA}_{1 \mathrm{c}}$ concentration $(r=-0.41, p=0.001)$

increased incidence of asymptomatic ischemia, myocardial infarction, and decreased likelihood of survival after myocardial infarction [10-12]. The autonomic nervous system closely integrates vital processes such as heart rate, blood pressure and myocardial contractility and as a consequence plays a pivotal role in the regulation of the cardiovascular system. Summarized data of nine studies showed that after 5.8 years, mortality in diabetic patients with CAN was $29 \%$, while it was $6 \%$ in those without CAN [7]. Myocardial infarction is the primary cause of death among diabetic patients. A large longitudinal study proved the initial evidence that HRV was a powerful predictor of cardiac mortality after myocardial infarction [13]. The vagus nerve plays a crucial role in the mediation of HRV. The ability to augment vagal activity can be quantified by baroreflex sensitivity. The ATRAMI study (Autonomic Tone and Reflexes After Myocardial Infarction) confirmed that after myocardial infarction, the analysis of baroreflex sensitivity has significant prognostic value independently of left ventricular ejection fraction and ventricular dysrhythmia. It significantly adds to the prognostic value of HRV [14]. It has also been shown by analyzing HRV and baroreflex sensitivity that

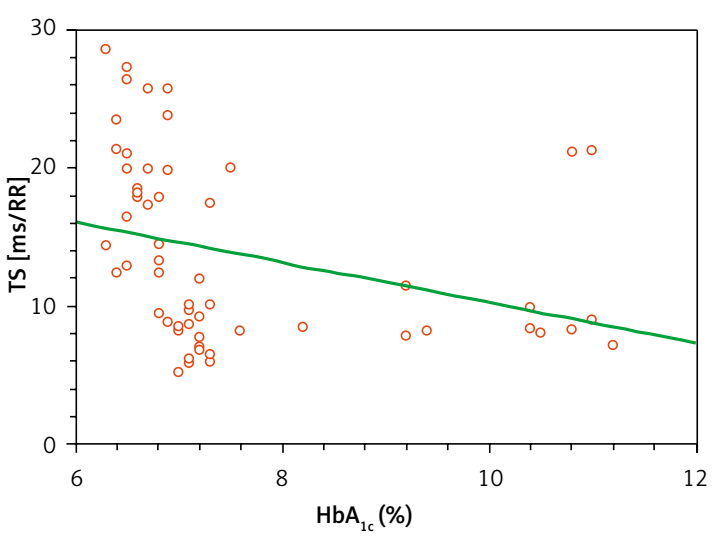

Figure 4. Correlation between TS (turbulence slope) and $\mathrm{HbA}_{1 \mathrm{c}}$ concentration $(r=-0.31, p=0.02)$

cardiovascular adaptation mechanisms are severely impaired in patients with long-term type 1 diabetes [15]. Baroreflex can be evaluated using HRT, which is a relatively new and simple method, representing a reliable indicator of baroreceptor sensitivity $[2,3]$. Our study revealed that both HRT parameters - TO and TS - were found to be significantly improved in patients with better glycemic control. This observation is consistent with other studies stating that glycemic control is an important factor in the pathogenesis of CAN [16-19].

Ewing's battery tests are reliable tests commonly used to detect CAN [20], though their application is limited, because they are very time consuming and require patient compliance, and they are another method of measurement of HRV. A decrease in HRV is the earliest sign of CAN; therefore a beat-to-beat HRV is suggested as a useful diagnostic test by the American Diabetes Association [1].

In our study, HRT was used for assessing the autonomic nervous system in DM instead of HRV. There are several papers presenting changes of HRV in diabetic patients. Also, Ewing's battery tests are based on RR variability. On the other hand, there are many different HRV parameters that can be analyzed in the time domain (e.g. SDNN, SDANN, SDNNI, pNN50, RMSDD) and in the frequency domain (e.g. LF, HF, HF/LF ratio). Until now, there is no consensus in which HRV parameters and cut-off values should be used for clinical practice. In this study, only SDNN significantly correlated with $\mathrm{HbA}_{1 \mathrm{c}}$. According to our knowledge, using HRT may be as useful as HRV analysis. Konduracka et al. [5] found significantly decreased TS but essentially similar TO values in type 1 diabetic patients in comparison with healthy controls. Balcioglu et al. [4] also reported decreased TS but similar TO values in type 2 DM with and without CAN. In our study, we did not assess the presence of CAN, but only used HRT for monitoring changes of autonomic nervous system function during 
intensive insulin therapy. HRT tests are not standardized for detection of CAN and have no cut-off values for diagnosis of CAN; for that reason HRT parameters may be particularly useful in monitoring changes of baroreceptor sensitivity in patients with DM. Heart rate turbulence may be useful in monitoring changes of autonomic nervous system functions in patients with DM instead of HRV parameters or Ewing's tests. This finding needs to be verified by larger studies.

The major limitation concerning the measurement of HRT is that sinus rhythm and the presence of ventricular premature beats are mandatory for its evaluation. Another limitation is the small study group.

In conclusion, intensive insulin therapy, leading to better glycemic control, results in improvement of HRT. Heart rate turbulence parameters may be a useful monitoring tool for the function of the autonomic nervous system in patients with diabetes mellitus.

\section{References}

1. Boulton AJM, Vinik Al, Arezzo JC, et al. Diabetic neuropathies - A statement by the American Diabetes Association. Diabetes Care 2005; 28: 956-62.

2. Guzik P, Schmidt G. A phenomenon of heart-rate turbulence, its evaluation, and prognostic value. Card Electrophysiol Rev 2002; 6: 256-61.

3. Lin LY, Lai LP, Lin JL, et al. Tight mechanism correlation between heart rate turbulence and baroreflex sensitivity: sequential autonomic blockade analysis. J Cardiovasc Electrophysiol 2002; 13: 427-31.

4. Balcıoglu S, Arslan U, Türkoglu S, Özdemir M, Çengel A. Heart rate variability and heart rate turbulence in $\mathrm{pa}$ tients with type 2 diabetes mellitus with versus without cardiac autonomic neuropathy. Am J Cardiol 2007; 100: 890-3.

5. Konduracka E, Pierucha AZ, Galicka-Latala D, et al. Analysis of heart rate turbulence in patients with 1st type diabetes mellitus. Europace 2013; 4 (Suppl. A): 41.

6. Vinik Al, Maser RE, Mitchell BD, Freeman R. Diabetic autonomic neuropathy. Diabetes Care 2003; 26: 1553-79.

7. Ziegler D. Diabetic cardiovascular autonomic neuropathy: prognosis, diagnosis and treatment. Diabetes Metab Rev 1994; 10: 339-83.

8. Bauer A, Malik M, Schmidt G, et al. Heart rate turbulence: standards of measurement, physiological interpretation, and clinical use: International Society for Holter and Noninvasive Electrophysiology Consensus. J Am Coll Cardiol 2008; 52: 1353-65.

9. Vinik Al, Maser RE, Mitchell BD, Freeman R. Diabetic autonomic neuropathy. Diabetes Care 2003; 26: 1553-79.

10. Langer A, Freeman MR, Josse RG, Steiner G, Armstrong PW. Detection of silent-myocardial-ischemia in diabetes-mellitus. Am J Cardiol 1991; 67: 1073-8.

11. Roy TM, Peterson HR, Snider HL, et al. Autonomic influence on cardiovascular performance in diabetic subjects. Am J Med1989; 87: 382-8.

12. Ziegler D. Cardiovascular autonomic neuropathy: clinical manifestations and measurement. Diabet Rev 1999, 7: 342-57.
13. Kleiger RE, Miller JP, Bigger JT, Moss AJ. Decreased heart-rate-variability and its association with increased mortality after acute myocardial-infarction. Am J Cardiol 1987; 59: 256-62.

14. La Rovere MT, Bigger JT, Marcus FI, Mortara A, Schwartz PJ. Baroreflex sensitivity and heart-rate variability in prediction of total cardiac mortality after myocardial infarction. Lancet 1998; 351: 478-84.

15. Lengyel C, Torok T, Varkonyi T, Kempler P, Rudas L. Baroreflex sensitivity and heart-rate variability in insulin-dependent diabetics with polyneuropathy. Lancet 1998; 351: 1436-7.

16. Kempler P, Varadi A, Tamas G. Autonomic neuropathy in newly diagnosed diabetes-mellitus. Diabetes Care 1993; 16: 848-9.

17. Kempler P, Tesfaye S, Chaturvedi N, et al. Autonomic neuropathy is associated with increased cardiovascular risk factors: the EURODIAB IDDM Complications Study. Diab Med 2002; 19: 900-9.

18. Witte DR, Tesfaye S, Chaturvedi N, Eaton SEM, Kempler P, Fuller JH. Risk factors for cardiac autonomic neuropathy in type 1 diabetes mellitus. Diabetology 2005; 48: 164-71.

19. Burger AJ, Weinrauch LA, D'Elia JA, Aronson D. Effect of glycemic control on heart rate variability in type I diabetic patients with cardiac autonomic neuropathy. Am J Cardiol 1999; 84: 687-91.

20. Ewing DJ, Martyn CN, Young RJ, Clarke BF. The value of cardiovascular autonomic function-tests -10 years experience in diabetes. Diabetes Care 1985; 8: 491-8. 